\title{
Human Friendship Estimation Model for Communication Robots
}

\author{
Takayuki Kanda $^{1}$, Shogo Nabe ${ }^{1 \& 2}$, Kazuo Hiraki $^{1 \& 2}$, Hiroshi Ishiguro ${ }^{1 \& 3}$, and Norihiro Hagita ${ }^{1}$ \\ ${ }^{1}$ ATR ${ }^{2}$ The University of Tokyo ${ }^{3}$ Osaka University
}

\begin{abstract}
Based on the analysis of non-verbal inter-human interaction, this paper proposes a model for estimating human friendships in the presence of a humanoid robot. Our previous study in an elementary school provided rich video data of two months of interhuman interaction in the presence of a humanoid robot. Such data are particularly useful for developing a robot's social ability: a friendship estimation capability. We analyzed the video based on an observation method to analyze the interaction among children and the robot. From their non-verbal interactions, several important factors for friendship estimation were retrieved, including touch, gaze, co-presence, and distance. Gender was also considered a factor in the model, since gender differences were observed in non-verbal interactions. The model discriminated between friendly and non-friendly relationships among the children with $74.5 \%$ accuracy for boys and $83.8 \%$ for girls.
\end{abstract}

\section{Author Keywords}

\section{Communication robot, friendship estimation, field trial, video analysis}

\section{INTRODUCTION}

Recent developments in the field of robotics have ushered us to the point where interactive robots are used in socially assistive applications. Shibata et al. developed a seal-like robot (Paro) for therapeutic purposes and demonstrated its social effect for encouraging communication among inpatients and caregivers (2001). Kozima et al. placed a creature-like remote-controlled robot (Keepon) in a daycare for developmental disordered children to encourage social behaviors (2005).

We believe that schools will be important fields for socially assistive robots because children sometimes have problems making friends, which is connected to bullying. Thus, robots must be able to recognize human relationships for such socially assistive applications in practical situations. The final goal of these socially assistive applications is to support users (e.g., children in schools, senior inpatients at care centers, etc.) to establish socially rich relationships with others and help prevent such problems as bullying in classrooms, which might become one important role for robots.

For instance, some cases in our previous research suggested the importance of recognizing the relationships children have with other children in socially assistive applications. We conducted a preliminary study where "Robovie,” our communication 


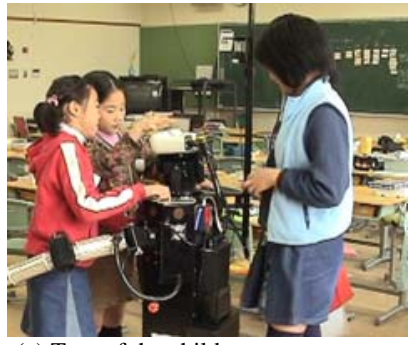

(a) Two of the children are close friends

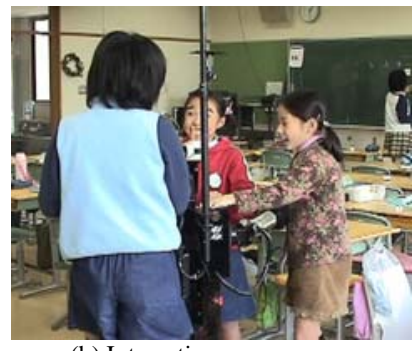

(b) Interaction

triggered by robot

Fig. 1 Inter-human interaction in robot's presence

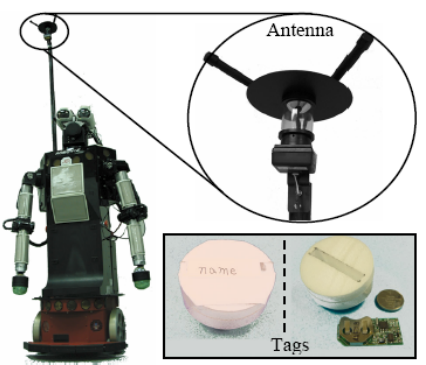

Fig. 2: Robovie and Wireless tags

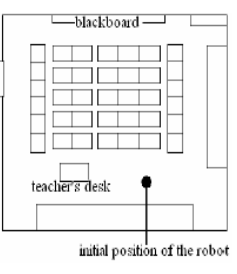

Fig. 3: Environment of the elementary school

robot, interacted with elementary school children for two months (Kanda et al. 2004a). One day during the experiment, we observed three girls playing with Robovie (Fig. 1). The two girls who were friends stuck together and often exchanged glances with smiles while playing with the robot. But the third girl kept acting toward the robot as though following the lead of the other two. She touched the robot and spoke to it in the same way as the other two girls without actually interacting with them. However, this situation changed when the robot started a game that greatly interested all three and that involved interaction together. In this case, the robot could have mediated the friendships among the children, if it had autonomously recognized these relationships. During this case, the robot performed additional behaviors to trigger interaction involving all three (as it actually did once by chance.) For longer terms of interaction, by recognizing relationships among children, the robot might provide chances to enrich their relationships. For example, the robot could have said, "Let's play a game. How about asking (child's name) to play? Please call her over here.”

In this paper we analyzed the video data obtained in the preliminary study and established a model for socially assistive robots to recognize friendships among children from non-verbal interaction. The video data were analyzed with an observation method, which is firmly established in psychology. Manually coding the data enables us to take an exploratory approach toward qualitative data and to quantify the results. Although this method requires manual data operation and is not immediately applicable to robot development, we hope that this study will play the same role as a similar case in psychology, such as the Facial Action Coding System (FACS) in image data processing. Developed by Ekman et al. 1978, FACS described facial expressions by focusing on muscle actions and presented them through quantified data. Our approach toward the video footage is identical; by quantifying the data with the observation method, we reveal crucial factors in recognizing human friendship. Our model estimates friendships among children from such simple non-verbal interactions as touching, gazing, and the distance maintained between each other. Even though currently the model needs the quantified data to be processed manually, we believe our model provides valuable findings to develop robotic software for social abilities. 


\section{BACKGROUND AND RELATED WORKS}

We believe that using robots to estimate children friendships is beneficial. As shown in Figure 1 , a robot has a physical presence in the same space in which humans interact. Since robots are often about the size of a child, they are large enough to invite multiple persons to interact together. Moreover, with such interactive behavior, they elicit various non-verbal behaviors including smiling, touching, gazing, simultaneous approaching, and withdrawing. They can also be equipped with sensors to capture physical interactions. Robots in the near future will be able to know who was present with whom, what they did together, and whether they interacted similarly or differently with the robot. Although we are still determining how to use this diverse information, we believe that it can provide more powerful ways of recognizing human relationships that benefit socially assistive robots.

One requirement exists for developing a relationship-recognition function for socially assistive robots: they must recognize relationships among people from interaction observed through sensory devices. We human beings can often infer relationships among others from behavior, but we are not so sure how we do this; in contrast, in developing a function for robots, describing this process of intuitive human ability at the level of sensory information is a necessary challenge. In other words, to make this available for robots, we only deal with directly observable behaviors and human gestures without considering the motivations and interpretations behind these behaviors.

Existing studies still do not provide enough knowledge to satisfy the requirements. The field of psychology contains a number of works that investigate human behavior and their relationships, but they are often difficult to apply to robot development. For example, Ladd et al. pointed out the behavioral differences between children who are likely to have positive or negative nominations as a friend by their classmates (1990). The study revealed that those who are nominated as a friend are likely to behave “cooperatively” toward classmates while the others behaved "roughly;” of course, such behavioral categories would be difficult to be addressed by robot sensors. One psychology study that helps our purpose is Hall’s work that investigated the distance between people in their relationships (1996). Distance is a proper feature, since we can deal with it through sensors. We must identify such a useful feature to estimate people’s relationships.

Studies can also be found in computer science that investigate human behavior and their relationships, though we consider that a large space still remains to be investigated for robots. Eveland et al. analyzed online communication on a computersupported collaborative work (CSCW) system (1986). They plotted the data for all users on a sociogram based on the amount of online communication among them. Nomura et al. developed a Web-analyzing system to retrieve human online relationships from hyperlinks in their web pages (2002). Regarding real-world human relationships, Choudhury et al. studied the visualization 
of the amount of face-to-face conversation using wearable sensors (2003). However, their study only used the utterances of people detected by wearable sensors on people, which is still too hard for a robot to recognize by its own sensors. Thus, none of these previous computer science studies are applicable for a socially assistive robot to estimate human relationships in the realworld.

To determine what behavioral information we can use, in this study we utilized the video data obtained from a field trial and observed both human-human and human-robot interaction (details in Section 4). We consider this an efficient approach for developing socially assistive robots. Video obtained in field trials represents a rich deposit of information on interaction among humans and robots that we cannot get from controlled experiments in laboratories. However, since these are qualitative data, retrieving solid findings is difficult. Our purpose was to utilize this rich data source to develop socially assistive robots, in particular, to obtain a model that recognizes friendly human relationships from interaction.

\section{FIELD TRIAL}

This section briefly describes the two-month field trial we conducted (Kanda et al. 2004a) in an elementary school with "Robovie" the communication robot. We analyzed the obtained video data by observing human-robot interaction in this field trial. In addition, we tested our initial friendship estimation model.

\subsection{Robovie and Person Identification}

Figure 2 shows "Robovie," the communication robot (Ishiguro et al. 2003) used in this experiment, who is capable of human-like expression and recognizes individuals using various actuators and sensors. Its body is highly articulated to produce sufficient gestures for effective communication with humans. Sensory equipment, including auditory, tactile, ultrasonic, and vision sensors, and processing and motor control hardware, are located inside its body.

We installed a wireless tag system capable of multi-person identification to identify individuals. Recent radio frequency identification (RFID) technologies enable the use of contactless identification cards and chips in the field. In this study, children were given easy-to-wear nameplates (5 cm in diameter) in which a wireless tag was embedded. Each tag (Fig. 2, lower-right) periodically transmitted its ID to the reader installed on the robot, providing the robot with a robust means of simultaneously identifying many children.

\subsection{Interactive Behaviors for Long-term Interaction}

Robovie, who features a software mechanism for performing consistent interactive behaviors (Kanda et al. 2002), was designed 
to communicate at a child's level. One hundred interactive behaviors have been developed, seventy of which include such behaviors as shaking hands, hugging, playing rock-paper-scissors, exercising, greeting, kissing, singing, chatting, and pointing to a nearby object. Twenty are idle behaviors such as scratching the head or folding the arms, and the remaining ten are ambulatory behaviors. In total, the robot can utter more than 300 sentences and recognize about 50 words.

The interactive behaviors appeared as follows based on some simple rules. The robot sometimes triggered interaction with a child by saying “Let's play, give me your hand,” and exhibited idling or ambulatory behaviors until the child responded. Once the child reacted, Robovie continued performing friendly behaviors as long as the child responded. When the child stopped reacting, the robot stopped the friendly behaviors, said “good-bye,” and re-started idling or ambulatory behaviors.

Robovie’s interactive behaviors were prepared with three design principles for long-term interaction (Kanda et al. 2004a).

1. Calling children's names

In some interactive behaviors, the robot called a child's name if that child was within a certain distance. For instance, the robot would say, "Hello, Yamada, let’s play together” when Yamada approached the robot.

\section{Pseudo-development}

As a child's interaction with the robot increases, the robot displays more types of interactive behavior. For example, it shows at most ten behaviors to a child with whom it has never interacted. However, it may reveal up to 100 behaviors to a child with whom it has interacted for more than 180 minutes. Since the robot gradually changes interaction patterns to match each child's experience, the robot seems to learn from the interaction.

\section{Sharing secrets}

The robot shares its secret with children who have often interacted with it. We prepared a threshold of interaction time for each matter to motivate children who often played with the robot to engage in further interaction. Personal comments include “I like talking” (to a child who has played with it for more than 120 minutes), “I don’t like the cold” (180 minutes), and so forth.

\subsection{Experimental Settings}

Using this robot we performed a two-month experiment at an elementary school with a class of 37 fifth-graders (10-11 years old, 18 boys and 19 girls). The two-month experimental period actually comprised 32 experiment days. We put the robot in the classroom (Fig. 3), where the children could freely interact with it during a 30-minute recess after lunch. We distributed questionnaires about friendship before the experiment to examine the estimation model. In the questionnaires, each child supplied 
the names of a maximum of five friends in class. The interactions of children and the robot were video recorded by an external camera. This procedure was within ethical guidelines and was approved by a human subject IRB board.

\subsection{Results}

Since the video data from the experiment will be used later in this research, here we briefly give an overview of the experimental results (See (Kanda et al. 2004a) for more details). Figure 4 shows the transition of interaction with the children. The dotted lines separate the nine weeks that comprise the two-month period. About ten children interacted with the robot every day. The average interaction time of each child during the whole experiment was about 71 minutes; in the first two weeks the robot caused a lot of excitement, and then the number of interacting children gradually decreased, although another increase occurred in the last two weeks. We classified the nine weeks into three principal phases and explained the interaction transitions during the two months by describing these phases.

\section{First phase $\left(1^{\text {st }}-2^{\text {nd }}\right.$ weeks): Robovie created big excitement}

Children crowded around the robot on the first and second days (Fig. 5b); on the first day, at most 17 children simultaneously stayed around it. They even formed a line to play with it (Fig. 5c). During the first two weeks, it still seemed so novel to the children that someone was always around the robot, and the rate of vacant time was nearly 0 . But the number of gathered children gradually decreased.

\section{Second phase ( $3^{r d}-7^{\text {th }}$ weeks): Stable interaction to satiation}

Everyday about ten children approached the robot, and some played with it (Fig. 5a). When it was raining, children who usually played outside played with the robot, and so the number of children interacting with it increased. During these five weeks, the number of interacting children gradually decreased and vacant time increased. The "sharing of secret" behavior, which first

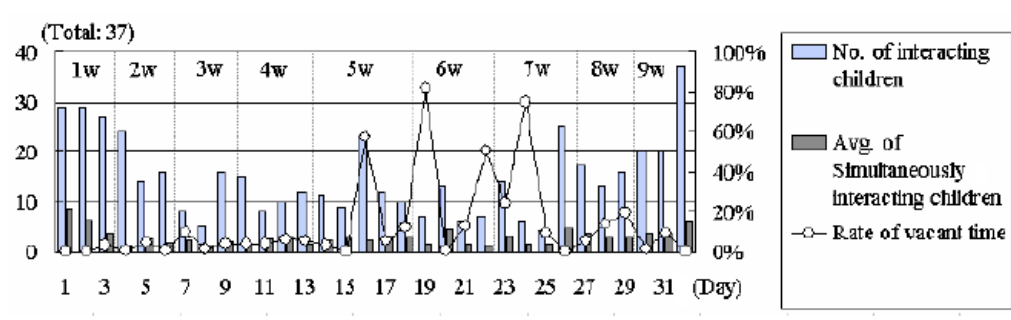

Fig. 4 Transitions of interaction between children and robot

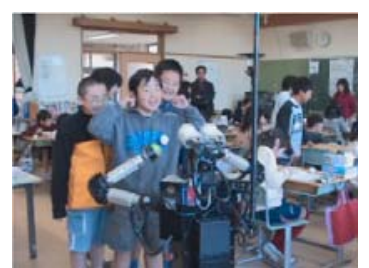

(a) Daily interaction

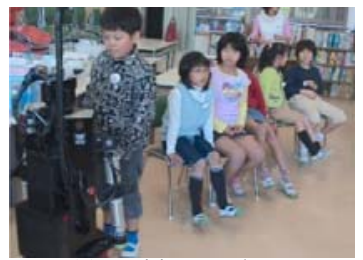

(c) Waiting to play

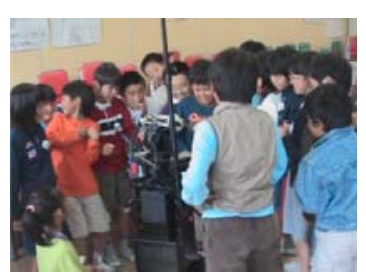

(b) Beginning of first day

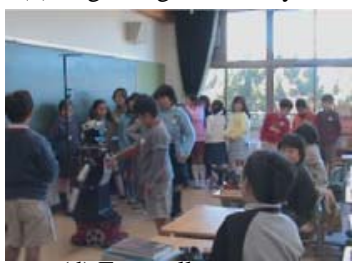

(d) Farewell party

Fig. 5 Scenes from experiment 
appeared in the fourth week, gained popularity.

The robot gradually performed new behaviors based on the pseudo-development mechanism, and these behaviors caught the children’s attention. For example, when the robot's eyes were covered, it brushed off this obstruction and complained, “I can’t see.” This new behavior was so popular that many children tried to cover the robot's eyes.

\section{Third phase ( $8^{\text {th }}-9^{\text {th }}$ weeks): Saying goodbye}

Even though the number of children who approached the robot increased during these two weeks, the number who played with it did not. Many simply approached and watched the interaction for a while. On the first day of the eighth week, the teacher told them that the robot would leave the school at the end of the ninth week. We believe the children were affected by this announcement and suggestion.

The "sharing secret" behavior became popular among the children, many of whom around the robot were absorbed in asking for its opinions. They made a list on the blackboard of the secrets they heard from the robot. One opinion, "I like the class teacher," was the children's favorite. After the robot said it, some children ran out of the classroom to tell their teacher. On the last day, the children held a farewell party for the robot and formed a line to play individually with it (Fig. 5d).

To analyze the video, we excluded several days from the recorded data. During the first five days, children crowded around the robot or formed a line to play with it. The class also held a farewell party for the robot on the experiment's last day. While the previous study included these days in the data for friendship estimation, this study excluded them to concentrate on more common interaction around the robot.

\section{FRIENDSHIP ESTIMATION MODEL}

\subsection{Friendship Definition}

In this study, we define the relationship between A and B as a "friendship" if either referred to the other as a friend on the distributed questionnaires, which is the same definition as our previous study (Kanda et al. 2004a; Kanda et al. 2004b).

Moreover, in this paper we only deal with the relationships between children of the same gender, since it is known in psychology that children of this age are most likely to have same-sex friends (McConnell and Odom 1986). Indeed, no cross-sex friendships were reported in our questionnaires. In addition, gender differences of friendship are found even among children less than 12 years old (Argyle and Henderson 1985). Since we aim to establish a model to estimate human relationships, we should not tackle such complicated cross-sex friendship problems in the early stages. We believe that estimating friendship within gender will be a starting point to estimate cross-gender relationships in future works. 
Table 1: Categories of interaction found in the pre-coding analysis of video data

\begin{tabular}{|l|l|}
\hline \multicolumn{2}{|c}{ pre-coding analysis of video data } \\
\hline Category & operational definition \\
\hline Simultaneous stay time & Two children observed at the same moment \\
\hline Simultaneous appearance & Two children appeared within 10 seconds \\
\hline Simultaneous withdrawal & Two children went away within 10 seconds \\
\hline Touch & $\begin{array}{l}\text { A child touched another one with his/her } \\
\text { palm } \\
\text { (Do not include touch by other parts of body) }\end{array}$ \\
\hline Gaze & Looking at a immediate neighbor's face \\
\hline Smile & Smiling to an immediate neighbor. \\
\hline Together & Two children separated by less than 50 cm. \\
\hline Approaching & $\begin{array}{l}\text { 'Together' condition is a prerequisite } \\
\text { One child moved his/her position toward the } \\
\text { other, while the other did not move his/her } \\
\text { position. } \\
\text { (not analyzed in coding) }\end{array}$ \\
\hline verbal interaction & (not analyzed in coding) \\
\hline interaction in context
\end{tabular}

\subsection{Preparation for the model}

In contrast with our previous study, the friendship estimation model in this paper exploits non-verbal interaction between friends. Our first approach observed the interaction between children around the robot to reveal what interaction is available for estimating friendship. This pre-coding enabled us to code the categorized interactions and to quantify them. Finally, we established a model to make estimations from the data and used the data collected from our field trial in the elementary school, described in II, in the following analysis.

\subsubsection{Pre-coding: reveals interaction among children}

\section{Method}

To reveal what kinds of interaction are available for friendship estimation, we conducted a preliminary analysis based on the video data recorded during the experiment by an external camera. We focused on individual children and described each action they made. We selected a minute of footage for each of six randomly selected subjects (three boys and three girls), who interacted with the robot in the presence of other children. The subjects were selected in the order they appeared on the video during the experiment, starting from a day in the middle of two months of the recorded data $\left(17^{\text {th }}\right.$ day), to exclude outlier data, where the subjects paid excessive attention to the robot due to its novelty. Analysis was done by describing all the "actions" and the "location toward the robot and other children" observed in the subjects.

\section{Results}

We obtained the following categorized interaction from this analysis (Table 1, Fig. 6):

'Simultaneous stay': two children simultaneously remain around the robot (friendship estimation model of our previous study (Kanda et al. 2004a) also used this information). 
'Simultaneous appearance': two children approach together to play with the robot.

'Simultaneous withdrawal': two children move away from the robot together.

'Together': two children position themselves very close to each other.

'Approaching': a child moves toward another child.

'Gaze': a child looks at the face of another child.

'Smile': a child smiles at another child.

'Touch': a child intentionally touches another child.

'Verbal interaction': either one instance of speech, laughter, or other verbal action made in interaction between two children.

'Interaction in context': Considered inter-human interaction due to context, for example, "a child touches the robot in the same place in the same way as another child just did.”

\subsubsection{Coding: qualifies non-verbal interaction}

\section{Method}

We quantified the non-verbal interactions among children in all the above categories by coding the video. That is, we coded 'simultaneous stay,' ‘simultaneous appearance,' ‘simultaneous withdrawal,' 'touch,' 'gaze,' 'gaze with smile,' 'together,' and ‘approaching' among the categories in Table 1 and excluded 'verbal interaction' and 'interaction in context' from our analysis. 'Verbal interaction' was excluded because the video data were often so noisy that we could not determine whether the children were uttering sounds. 'Interaction in context' was also excluded due to the difficulty of objectively exploiting it.

Interval coding was done with a 1/0 sampling method (Kaiho et al. 2006; Eysenck et al. 1972) by sampling the children's presence and their positions compared to the robot's position every ten seconds. During these ten seconds, if a 'touch' or a 'gaze' was observed, it was recorded (with its expression for 'gaze' too). Operational definitions of each non-verbal interaction are shown in Table 1. For 'gaze' data, we only counted gazes between two children located next to each other, because in the other cases it was difficult to decide whether a gaze was directed toward a particular child or toward something else (e.g., the robot was between the two).

For these analyses, we first established the coding scheme by observing interaction among children and then involved a coder blinded to their relationships. We only used one coder since the amount of video data was large while we expected a degree of coding reliability. We estimated reliability by choosing three days of video data among 26 and compared the coded results 
Table 2: Observed number and occurrence frequency of each non-verbal interaction (for all 37 children)

\begin{tabular}{|c|c|c|c|c|c|c|c|c|c|c|c|c|c|c|c|c|}
\hline \multirow{2}{*}{$\begin{array}{l}\text { Friends/non-friends } \\
\text { Num. of times }\end{array}$} & \multicolumn{2}{|c|}{ Simul. stay } & \multicolumn{2}{|c|}{$\begin{array}{c}\text { Simul. } \\
\text { appearance }\end{array}$} & \multicolumn{2}{|c|}{$\begin{array}{c}\text { Simul. } \\
\text { withdrawal }\end{array}$} & \multicolumn{2}{|c|}{ Touch } & \multicolumn{2}{|c|}{ Gaze } & \multicolumn{2}{|c|}{ Smile } & \multicolumn{2}{|c|}{ Together } & \multicolumn{2}{|c|}{ Approaching } \\
\hline & 9660 & 11730 & 58 & 41 & 64 & 54 & 421 & 276 & 1431 & 1161 & 400 & 345 & 2058 & 1390 & 146 & 145 \\
\hline Occurrence freq. & --- & 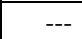 & --- & $\begin{array}{ll}-- \\
--\end{array}$ & --- & --- & 0.044 & 0.024 & 0.148 & 0.099 & 0.042 & 0.03 & 0.214 & 0.12 & 0.015 & 0.012 \\
\hline
\end{tabular}

Table 3: Weight of each non-verbal interaction $\left(\alpha_{i}\right)$ for friendship estimation models (for 25 children who interacted with robot over 10 minutes)

\begin{tabular}{|l|r|r|r|r|r|r|r|r|}
\hline & \multicolumn{1}{|c|}{$\begin{array}{c}\text { Simul. } \\
\text { stay time }\end{array}$} & $\begin{array}{c}\text { Simul. } \\
\text { appearance }\end{array}$ & $\begin{array}{c}\text { Simul. } \\
\text { withdrawal }\end{array}$ & Touch & Gaze & Smile & Together & Approaching \\
\hline Both genders & $\mathbf{1 . 1 3}$ & 0.24 & 0.16 & $\mathbf{0 . 4 0}$ & 0.15 & -0.13 & 0.28 & $\mathbf{- 0 . 3 0}$ \\
\hline male & 0.36 & -0.12 & $\mathbf{0 . 6 3}$ & $\mathbf{- 0 . 4 4}$ & $\mathbf{1 . 3 3}$ & -0.23 & 0.15 & -0.22 \\
\hline female & $\mathbf{2 . 1 0}$ & 0.25 & -0.29 & $\mathbf{0 . 5 2}$ & -0.18 & 0.31 & 0.50 & $\mathbf{- 0 . 6 7}$ \\
\hline
\end{tabular}

Table 4: Performance of friendship estimation model

\begin{tabular}{|l|r|r|}
\hline & Simul. time & All interaction \\
\hline Both gender & $65.0 \%$ & $71.3 \%$ \\
\hline Male & $50.9 \%$ & $74.5 \%$ \\
\hline Female & $73.3 \%$ & $83.8 \%$ \\
\hline
\end{tabular}

with that of another person. As averages of the eight categories, 92.7\% of their coding matched, and the kappa value was 0.556 , which represents marginal matching.

\section{Results}

Table 2 shows the observed number and the occurrence frequency of each category of non-verbal interaction among friends and non-friends. We analyzed all the data involving the 37 children. 'Occurrence frequency' is the rate of the occurrence of a particular interaction category within ten seconds of 'simultaneous stay' between two children; thus it is only defined for 'touch,' 'gaze,' 'smile,' 'together,' and 'approaching.' (Note that 'simultaneous appearance' and 'simultaneous withdrawal' are not interactions that occur during simultaneous stay.)

These results suggest that the categories enable us to estimate friendship from each of them. 'Simultaneous appearance' and 'simultaneous withdrawal' were observed just as often among friends as non-friends. Since there are, however, twice as many non-friendly relationships as friendly ones, the results suggest children preferred to appear or withdraw with friends rather than with non-friends. For the categories where occurrence frequency could be defined, the results suggest a high estimation performance if the values score relatively high for 'friends' compared with 'non friends,' which actually was the case.

In the data, pairs of girls were observed nearly four times as often as pairs of boys because girls generally had longer interaction with the robot (see Kanda et al. 2004a).

\subsection{Friendship Estimation Model}

From these results we designed a model to estimate friendships that used all the non-verbal interactions coded above. 
We made the model to estimate the friendships out of children who interacted with the robot for more than 10 minutes (10 boys and 15 girls). This was unavoidable since no model can estimate friendship between people who never appear within the range of observation. There were 51 friendships among these children.

Moreover, we estimated the same-gender friendships for both the male and female participants. Thus, this study focused on the estimation of 51 friendships (17 male-friendships and 34 female-friendships) out of 160 possible relationships (55 for boys and 105 for girls).

In the model, the estimated friendship between children $A$ and $B$ was defined as:

$$
\begin{aligned}
& \text { Friend }(A, B)=i f\left(\text { Friendliness }(A, B)>F_{T H}\right) \\
& \begin{aligned}
& \text { Friendliness }(A, B) \\
&=\sum_{i} \alpha_{i} * \text { Interaction }_{i}(A, B) \\
&= \alpha_{\text {simul.stay }} * \text { Interaction }_{\text {simul.stay }}(A, B) \\
&+\alpha_{\text {simul.appearance }} * \text { Interaction }_{\text {simul.appearance }}(A, B) \\
&+\alpha_{\text {simul.withdrawal }} * \text { Interaction }_{\text {simul.withdrawal }}(A, B) \\
&+\alpha_{\text {touch }} * \text { Interaction }_{\text {touch }}(A, B) \\
&+\alpha_{\text {gaze }} * \text { Interaction }_{\text {gaze }}(A, B) \\
&+\alpha_{\text {smile }} * \text { Interaction }_{\text {smile }}(A, B) \\
&+\alpha_{\text {nearbying }} * \text { Interaction }_{\text {nearbying }}(A, B) \\
&+\alpha_{\text {approaching }} * \text { Interaction }_{\text {approaching }}(A, B)
\end{aligned}
\end{aligned}
$$

where $i$ corresponds to each non-verbal interaction, $F_{t h}$ is a threshold, $\operatorname{Interaction}_{i}(A, B)$ is a function that gives a score based on the interaction components between $A$ and $B$, which is normalized based on the expected occurrence rate for each child, and $\alpha_{i}$ is a weight for each interaction to be considered in the model, which is the most essential part of this estimation. Since automatically identifying the appropriate value of $F_{t h}$ is not easy, for calculating discrimination accuracy later (Table 4), we manually adjusted threshold $F_{t h}$ so that Equation (1) performed the best estimation. The results for various values $F_{t h}$ are shown in Fig. 7.

\section{ESTABLISHED MODEL FOR FRIENDSHIP ESTIMATION}

\subsection{Model}

We conducted discrimination analysis on the coded data to obtain the best $\alpha_{i}$ values (in (2)) and to discriminate the relationships among these particular data, though it remains uncertain whether these parameters are applicable to other data. Our aim is to establish a model that improves friendship estimation in certain settings. We consider that the performance of the model will reach its full potential when the best way for piecing together information from various non-verbal interactions is known. We chose three parameter sets that are expected to perform best in estimating friendships between males, females, and both. 


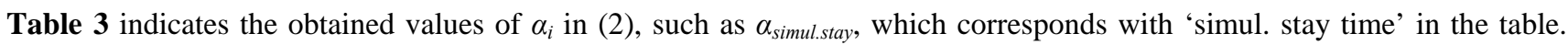
These results show which particular non-verbal interaction contributed to the estimation. Since each category of interaction has its data normalized, the values of $\alpha_{i}$ represent the meaningfulness of the corresponding information.

These results show the contributing categories in friendship estimation, where 'gaze,' 'simultaneous withdrawal,' and 'touch' were the three most meaningful categories for boys and 'simultaneous stay,' 'approaching,' and 'touch' for the girls. The categories with negative values only contribute to friendship estimation when some interaction of another category has also occurred at high frequency in every possible relationship (e.g., girls who are often close but seldom approach each other are likely to be friends). However, it remains unclear how these categories are linked, and revealing this is a challenge for future work.

\subsection{Estimation Result}

We applied two measures to evaluate our friendship estimation model. One way to measure the model's performance is: discrimination accuracy $=\frac{\text { number of relationships (both friendship and non }- \text { friendship) estimated correctly }}{\text { total number of possible relationships }}$,

which we define as 'discrimination accuracy.' However, this measure does not work appropriately when the number of friendships is relatively small compared to all possible relationships. For example, if we consider all possible relationships in a class, including male-female relationships, then based on the questionnaire responses, there would be 106 friendships among 666 possible relationships. If we suppose a classifier that categorizes every possible relationship as a non-friendship, the discrimination accuracy would be $84.1 \%$, meaning the evaluation is completely useless.

Instead, our previous study (Kanda et al. 2004a) proposed a second measure that focuses on the relevance of estimation ('coverage’ and 'reliability'). This measure is defined as:

$$
\begin{aligned}
& \text { reliability }=\frac{\text { number of friendships estimated correctly }}{\text { number of friendships estimated }} \\
& \text { coverage }=\frac{\text { number of friendships estimated correctly }}{\text { number of friendships from questionnaire }}
\end{aligned} .
$$

In this study, since the number of existing friendships is not as small as the number of possible relationships, applying both 'discrimination accuracy' and 'coverage and reliability' measures is possible. We applied the former method to evaluate the model as a whole and the latter to observe the trade-off between coverage and reliability in the model. 

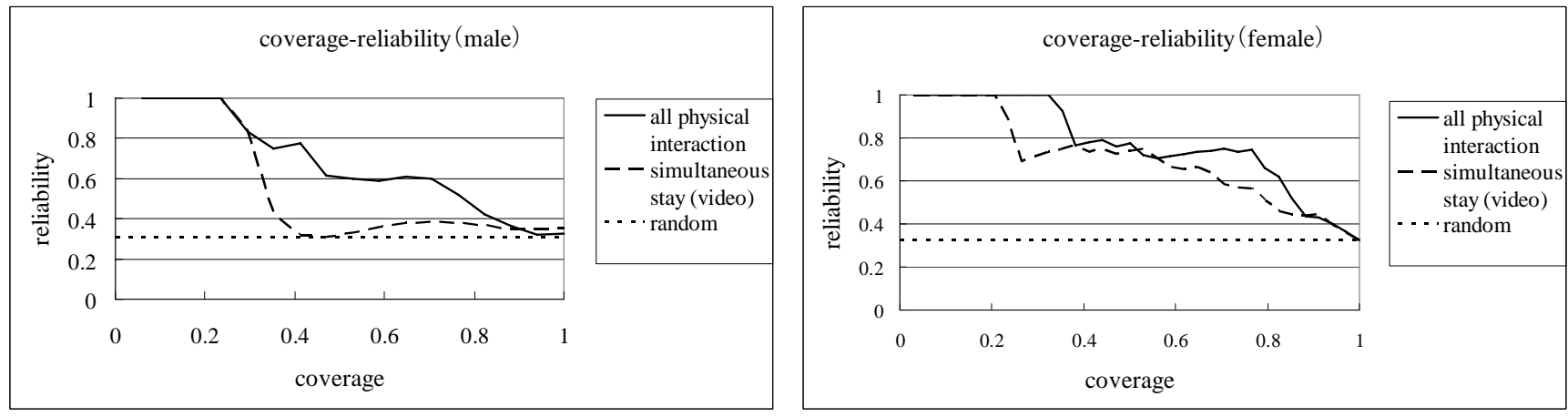

Fig. 7 Illustrations of friendship estimation results

Table 4 and Fig. 7 indicate the estimation results using two models: the estimation model from our previous study, which only used the 'simultaneous stay' time (this study’s data were collected by video coding, not by RFID tags), and the estimation model using all factors of the non-verbal interactions coded from the video. As mentioned in the method described in Section 5.1, both models have three sets of parameters: for 'male', for 'female,' and for 'both genders' (note that 'both genders' only counts friendships and possible relationships within the same gender, as mentioned in Section 4.1).

Table 4 indicates the discrimination accuracy of the established models. In this comparison, the value of the threshold of model $F_{t h}$ (in (1)) was set to guarantee the best performance from the parameter set. The model that used all the factors of nonverbal interactions coded from the video had a discrimination accuracy of $71.3 \%$, showing $6.3 \%$ improvement from the previous model that only used 'simultaneous stay.' This improvement was more remarkable when the model estimated friendships within the same gender. In this case the model's discrimination accuracy increased by $23.6 \%$ for males and $10.5 \%$ for females. This result suggests that using non-verbal interaction in friendship estimation is efficient and that performance improves by considering gender differences.

Figure 7 shows the coverage-reliability chart. Here, random represents the reliability of random estimation, assuming that all possible relationships are friendships (this represents the lower boundary of estimation). Obviously a trade-off exists between reliability and coverage that is controlled by $F_{t h}$. Non-verbal interactions improved the estimation of friendships within gender, given that the model successfully estimated $20 \%$ of the friendships with nearly $100 \%$ accuracy and $50 \%$ at accuracies between $60-80 \%$.

\section{DISCUSSION}

\subsection{Contribution to Socially Assistive Robotics}

\subsubsection{Friendship estimation model}


In this study, we established a model for estimating children's friendships in the presence of a humanoid robot. The established model included 'gaze,' ‘simultaneous withdrawal,' and ‘touch’ factors for estimating male friendships and 'simultaneous stay,' ‘approaching,' and ‘touch’ for estimating female friendships. Our model discriminated between friendly and non-friendly relationships among children with $74.5 \%$ accuracy for boys and $83.8 \%$ for girls. This represents a respective $23.6 \%$ and $10.5 \%$ improvement compared with our previous model that just utilized 'simultaneous stay' information. In the previous study, we used 'simultaneous stay' because it was the only available information from RFID tags. However, there is more information that a robot can potentially use; thus, in this study, we included such additional non-verbal information as 'gaze,' 'simultaneous withdrawal,' 'touch,' 'simultaneous stay,' 'approaching,' and 'touch.' From this study, we confirmed that the previous study used one important factor ('simultaneous stay') and revealed that we can achieve better estimation by including other non-verbal information.

Note that the children's friendships may change during the trial period. The robot was not only used for observing non-verbal behavior among children but also for interacting with them. There could be side effects since the robot was used as a mediator to form new friendships. It sometimes accidentally mediated children's relationships, as shown in the introduction's example, which might have affected estimation performance. Friendship questionnaires were completed before the experiment while observation was achieved through the experiment. Even though this effect is not so large, since the robot was not designed to mediate it, it might have influenced the estimation model's performance; for example, the model might perform slightly better than the results shown.

\subsubsection{Applicability for socially assistive robots}

We believe that elementary schools will be one potential working place for future interactive robots. They will serve as friends and guards that monitor classroom problems. One type of potential classroom problem is outside intrusions of suspicious people, during which a robot can function as a guard.

Another type of problem involves actions insider the classroom: bullying. This study suggests communication robot applications in a classroom situation for such problems. We believe that the established model enables us to develop an autonomous robot capable of estimating people’s friendships. Our vision for the utilization of this estimated information is that class teachers will monitor the estimated results to supplement their own class observations. Moreover, a robot might behave as a relationship mediator among children, as shown in the introduction's example. This mediator role could be expanded to include applications to bullying problems. We do not know to what extent we can make this application completely autonomous; but, at least, if a teacher specifies a target child for monitoring, the robot can expand that child's chances to interact with other children. In other words, mediation emanates from peers, not supervisors or authority figures, which offers teachers an additional 
intervention method. If teachers excessively intervene by themselves, sometimes bullying might disappear from the teachers' observable range while continuing outside the teachers’ observation.

In addition to such direct uses, we believe that the approach itself is beneficial for socially assistive robots. In many cases such human communication knowledge should be parameterized for implementation. This paper revealed a positive perspective of one straightforward approach for utilizing psychological methods and findings for engineering implementations.

\subsection{Ethical considerations}

When applying the friendship estimation technique to socially assistive applications, we must be very careful about ethical issues, which will mainly be caused by (1) incomplete estimation and (2) incorporating “a robot” into human-communication.

We should be aware that the model sometimes incorrectly estimates a possible relationship. For instance, a robot might describe a child as isolated (without friends); such an incorrect opinion might become truth simply because the robot said it, and the robot's statement might even initiate an actual bullying problem. Even if the estimation were correct, a child might be unhappy about such a public categorization. We suggest using such estimation results about problems and negative situations under human supervision. A robotic system accumulates well a large amount of data for many children, but a human being must be responsible for the estimation results. For example, in a future application, a teacher might check the robot’s report and offer feedback.

The second issue is a more general problem involving all human-robot communication and socially assistive robots. For example, even if future robots can prevent bullying, they might cause a situation where humans themselves cannot solve a bullying problem without robotic support. This must be carefully considered when developing real applications so that humans do not excessively rely on robots; robots must remain in service roles.

\subsection{Limitations}

Since this research was only conducted with one particular robot, Robovie, and children in a single classroom, its generalities are limited. Indeed, generalizing such factors is very difficult. Instead, we believe that the importance of our findings are the following: (1) robots can theoretically estimate children's friendship in a classroom; (2) this can be accomplished with the reported process by retrieving such non-verbal interactions as 'gaze,' ‘simultaneous withdrawal,' and 'touch.' We expect that these findings will work in general. When developing a socially assistive robot with friendship estimation capability, we believe that we can adjust appropriate parameters for the robot with the reported method that will be similar if the robot offers similar interaction: both verbal and non-verbal, and sometimes attracting a couple of children to interact together. 


\section{CONCLUSION}

In this paper, we described our model for friendship estimation that will be used by socially assistive robots. It was established by analyzing the video data of longitudinal children-robot interaction. We revealed the importance of particular factors in non-verbal interaction between friends. There is a gender difference in children’s interaction: 'gaze,' ‘simultaneous withdrawal,' and 'touch’ were essential among boys, and 'simultaneous stay,' ‘approaching,' and 'touch' were imperative among girls. From these findings, our model discriminated friendly and non-friendly relationships among children with $74.5 \%$ accuracy for boys and $83.8 \%$ for girls, which represents a respective $23.6 \%$ and $10.5 \%$ improvement compared with the existing model. Although the model uses hand-coded data, it will be useful for developing the social ability of socially assistive robots in the future.

\section{ACKNOWLEDGMENT}

This research was supported by the Ministry of Internal Affairs and Communications of Japan. The authors wish to thank Associate Professor Naoki Saiwaki at Nara Women’s University for his valuable advice and helpful discussions.

\section{REFERENCES}

Argyle, M. and Henderson, M., 1985, The anatomy of relationships, Penguin books.

Choudhury, T. and Pentland A., 2003, Modeling Face-to-Face Communication Using the Sociometer, IEEE Int. Conf. on Ubiquitous Computing.

Ekman, P. and Friesen, W. V., 1978, Manual for the Facial Action Coding System and Action Unit Photographs, Consulting Psychologists Press.

Eveland, J. D. and Bikson T. K., 1986, Evolving electronic communication networks: an empirical assessment, Proceedings of the 1986 ACM conference on Computer-supported cooperative work, pp. 91-101.

Eysenck, H. J., Arnold W., and Meili, R., (eds), 1972, Encyclopedia of Psychology, Seabury Press.

Hall E. T., 1996, Hidden Dimension, Doubleday Publishing.

Ishiguro, H., Ono, T., Imai, M., and Kanda, T., 2003, Development of an interactive humanoid robot "Robovie"-An interdisciplinary approach, Robotics Research, Springer, pp. 179-191.

Kaiho, H. and Kusumi, T. (eds), 2006, Encyclopedia of psychology (in Japanese), Asakura publishing.

Kanda, T., Ishiguro, H., Imai, M., Ono, T., and Mase, K., 2002, A constructive approach for developing interactive humanoid robots, IEEE/RSJ Int. Conf. on Intelligent Robots and Systems, pp. 1265-1270.

Kanda T., Sato, R., Saiwaki, N., and Ishiguro, H., 2004a, Friendly social robot that understands human's friendly relationships, Proc. IEEE/RSJ Int. Conf. on Intelligent Robots and Systems, pp. 2215-2222. 
Kanda, T. and Ishiguro, H., 2004b, Reading human relationships from their interaction with an interactive humanoid robot, Int. Conf. on Industrial and Engineering Applications of Artificial Intelligence and Expert Systems (IEA/AIE).

Kozima, H., Nakagawa, C., and Yasuda Y., 2005, Interactive robots for communication-care: A case-study in autism therapy, IEEE International Workshop on Robot and Human Interactive Communication (ROMAN-2005), pp. 341-346.

Ladd, G. W., Price, J. M., and Hart, C. H., 1990, Preschooler’s behavioral orientations and patterns of peer contact: predictive of peer status?, in Peer rejection in childhood, edited by S. R. Asher and J. D. Coie, Cambridge Univ. Pr., pp. 90-115

McConnell, S. R. and Odom, S. L., 1986, Sociometrics: Peer-referenced measures and the assessment of social competence, Children's social behavior, pp. 215-284.

Nomura S., Oyama S., Hayamizu T., and Ishida T., 2002, Analysis and Improvement of HITS Algorithm for Detecting Web Communities, The 2002 International Symposium on Applications and the Internet, pp. 132-140.

Scassellati, B., 2000, Investigating Models of Social Development Using a Humanoid Robot, Biorobotics, MIT Press.

Shibata, T. and Tanie, K., 2001, Physical and affective interaction between human and mental commit robot, Proc. IEEE Int. Conf. on Robotics and Automation, pp. 2572-2577. 